\title{
Henry E. Sigerist. Zum 50. Todestag
}

Marcel H. Bickel

Korrespondenz:

Prof. Dr. phil. Marcel H. Bickel

Medizinhistorisches Institut

Bühlstrasse 26

CH-3012 Bern
Am 17. März 1957 starb im Tessiner Dorf Pura Henry E. Sigerist, ein medizinischer Gelehrter und Organisator von internationalem Rang. Wer war Sigerist, und warum lohnt sich ein Rückblick 50 Jahre nach seinem Tod?

Als Sohn von Schweizer Eltern wurde Sigerist 1891 in Paris geboren, besuchte die Schulen in Paris und Zürich, begann ein Studium der Orientalistik in Zürich und London, vollendete ein Studium der Medizin in Zürich und München und wandte sich nach dem Staatsexamen 1917 in Zürich der Geschichte der Medizin zu. Er habilitierte sich daselbst und wurde 1925 als Nachfolger des Altmeisters Sudhoff auf den Lehrstuhl für Medizingeschichte in Leipzig berufen. 1932 folgte er einem Ruf an die Johns Hopkins University in Baltimore, als Nachfolger von William H. Welch und Direktor des ersten amerikanischen Instituts für Medizingeschichte. Schon in Leipzig erweiterte sich Sigerists anfänglich philologisch betriebene Medizingeschichte um den Einbezug ideengeschichtlicher, ökonomischer und sozialer Faktoren. In den zentralen 15 Jahren in Amerika wurde Sigerist zum unbestrittenen Meister seines Fachs, ein ungewöhnliches Phänomen im 20. Jahrhundert. Als Lehrer, Vortragender und durch seine Bücher erlangte er Berühmtheit. Medizingeschichte war für ihn nicht antiquarische Spezialität, sondern Mittel zum Verständnis der Medizin in ihrer Gesamtheit, insbesondere ihres sozialen Aspekts. Einer technisch entwickelten Medizin standen auch im reichsten Land der Erde schwerste Mängel einer bezahlbaren medizinischen Versorgung für alle gegenüber. So kämpfte Sigerist um Ziele wie Präventivmedizin, obligatorische Krankenversicherung, nationale Gesundheitssysteme bis hin zu sozialisierter Medizin nach sowjetischem Muster, die er an Ort und Stelle studiert hatte. In den 1930er Jahren war er mit seiner Abscheu vor dem Nationalsozialismus und mit einer sozialistischen Gesellschaftsutopie in durchaus respektabler intellektueller Gesellschaft. Das Ausbleiben konkreter Erfolge in der Organisationsform der medizinischen Versorgung hat ihn schliesslich wieder ganz in die Arme der Medizingeschichte getrieben. Eine mehrbändige Geschichte und Soziologie der Medizin sollte sein Lebenswerk krönen, und zu diesem Zweck legte er 1947 seine Professur in Baltimore nieder und zog sich nach

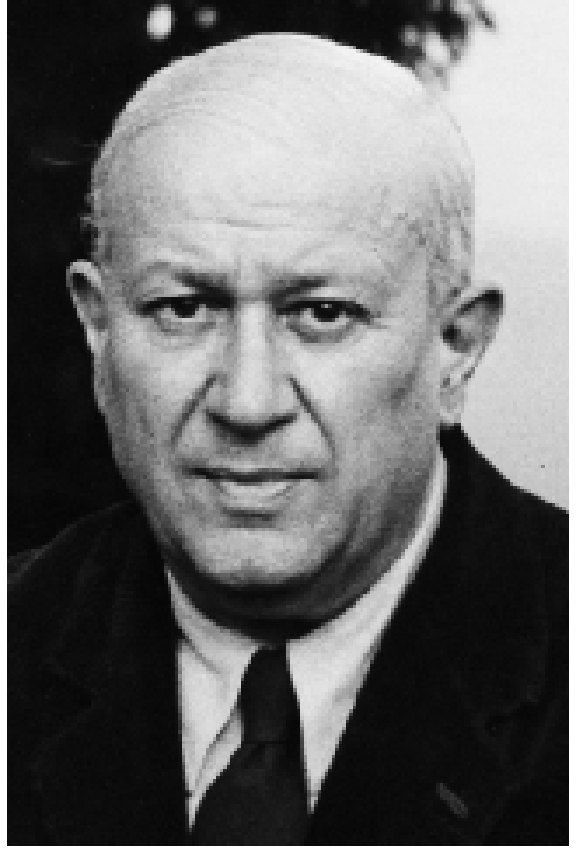

Henry E. Sigerist, 1891-1957

Pura im Tessin zurück. Die letzten zehn Jahren seines Lebens führten aus gesundheitlichen und anderen Gründen nur zu einem Anfang des geplanten Werks, allerdings zu einem vielversprechenden Beispiel einer medizinischen Historiographie unter neuen Gesichtspunkten.

Die hier skizzierte Biographie ist wohl bereits ungewöhnlich. Der Eindruck, den Sigerists Werk und wohl noch mehr seine Vielseitigkeit und seine Persönlichkeit gemacht hatten, führte in den zwei Jahren nach seinem Tod zu über 50 publizierten Nekrologen und Laudationes. Das alles mag in ähnlicher Form auch bei anderen bedeutenden Wissenschaftlern und Gelehrten zutreffen, die dann aber oft langsam in die Vergessenheit gleiten, da ihr Werk veraltet und vom Glanz neuer Sterne und Probleme überschattet wird. Was demgegenüber im Fall Sigerists erstaunt, ist die Tatsache, dass neue Generationen ihn noch lesen, sich mit seinem Werk beschäftigen und darüber schreiben. Über 130 Artikel und Buchkapitel von oft hohem wissenschaftlichem Niveau und Kritik sind seit seinem Tod erschienen, die Hälfte davon in den letzten 20 Jahren. Dies lässt aufhorchen! 
Als Medizinhistoriker wird Sigerist immer wieder erwähnt, nicht zuletzt als Begründer der modernen sozialhistorischen Richtung. Und die auch in den USA notwendige Diskussion um eine sozialverträgliche Gesundheitspolitik wird vielerorts, z.B. in der Vereinigung «Sigerist Circle», fortgeführt. An der Universität Leipzig wird 2007 ein Symposium über die Bedeutung von Sigerist 50 Jahre nach seinem Tod nachdenken. Den grossen Nachlass Sigerists verwalten die Archive der Universitäten Yale, Johns Hopkins, Zürich und Leipzig.

\section{Literatur}

- Becker C. Schriften über Henry Ernest Sigerist (1891-1957) - eine Bibliographie. In: Hahn S, Thom A, Herausgeber. «Kolloquium zum 100. Geburtstag von Henry Ernest Sigerist». Leipzig: Karl-Sudhoff-Institut; 1991. p. 37-45.

- Berg-Schorn E. Henry E. Sigerist (1891-1957). Köln: C.-E. Kohlhauer; 1978.

- Fee E, Brown TM, editors. Making Medical History. The Life and Times of Henry E. Sigerist. Baltimore/ London: The Johns Hopkins University Press; 1997.

- Miller G. A Bibliography of the Writings of Henry E. Sigerist. Montreal: McGill University Press; 1966.

- Sigerist Beeson N, Herausgeberin. Henry E. Sigerist. Autobiographische Schriften. Stuttgart: Georg Thieme Verlag; 1970. 